RUNNING HEAD: Social Ranking Effects on Tooth-brushing.

Social Ranking Effects on Tooth-brushing Behaviour.

John Maltby ${ }^{\mathrm{a} *}$, Kevin Paterson ${ }^{\mathrm{a}}$, Liz Day ${ }^{\mathrm{b}}$, Ceri Jones ${ }^{\mathrm{a}, \mathrm{c}}$, Hayley Kinnear ${ }^{\mathrm{a}}$ and Heather Buchanan $^{\text {d }}$

${ }^{a}$ College of Medicine, Biological Sciences, and Psychology, Henry Wellcome Building, Leicester University, Lancaster Road, Leicester, England, LE1 9HN.

b Department of Psychology, Sociology and Politics, Sheffield Hallam University, Sheffield, United Kingdom.

${ }^{\mathrm{c}}$ University Hospitals of Leicester NHS Trust, The Firs Glenfield Hospital , Leicester, LE3 9QP, United Kingdom.

${ }^{\mathrm{d}}$ Faculty of Medicine \& Health Sciences. Queen's Medical Centre, University of Nottingham, NG7 2UH, United Kingdom.

*Corresponding author. Tel.: +44 1162522482. E-mail address: jm148@le.ac.uk 
RUNNING HEAD: Social Ranking Effects on Tooth-brushing.

Social Ranking Effects on Tooth-brushing Behaviour. 


\begin{abstract}
Objective: A tooth-brushing social rank hypothesis is tested suggesting tooth-brushing duration is influenced when individuals position their behaviour in a rank when comparing their behaviour with other individuals.

Design: Study 1 used a correlation design, Study 2 used a semi-experimental design and Study 3 used a randomized intervention design to examine the tooth-brushing social rank hypothesis in terms of self-reported attitudes, cognitions and behaviour towards tooth-brushing duration.
\end{abstract}

Methods: Study 1 surveyed participants to examine whether the pereceived health benefits of tooth-brushing duration could be predicted from the ranking of each person’s tooth-brushing duration. Study 2 tested whether manipulating the rank position of the tooth-brushing duration influenced participant-perceived health benefits of tooth-brushing duration. Study 3 used a longitudinal intervention method to examine whether messages relating to the rank positions of tooth-brushing durations causally influenced the self-report tooth-brushing duration.

Results: Study 1 demonstrates that perceptions of the health benefits from tooth-brushing duration are predicted by the perceptions of how that behaviour ranks in comparison to other people’s behaviour. Study 2 demonstrates that the perceptions of the health benefits of toothbrushing duration can be manipulated experimentally by changing the ranked position of a person's tooth-brushing duration. Study 3 experimentally demonstrates the possibility of increasing the length of time for which individuals clean their teeth by focusing on how they rank among their peers in terms of tooth-brushing duration.

Conclusions: The effectiveness of interventions using social-ranking methods relative to those that emphasize comparisons made against group averages or normative guidelines are discussed. 


\section{Social Ranking Effects on Tooth-brushing behaviour}

According to the WHO Global Strategy report on oral health, 60 to 90 percent of schoolchildren and almost 100 percent of adults have dental cavities (World Health Organization, 2012).Key to the prevention of oral disease is proper tooth-brushing, with standard advice being to brush one's teeth twice a day, for at least two minutes (National Health Service, 2013), which is the key technique for plaque removal and anti-caries agent application (e.g. fluorides) (Sasahara \& Kawamura, 2000; Attin \& Hornecker, 2005), thereby reducing the risk of long-term tooth loss, periodontal disease, viral infections, and oral cancer (Broadbent, Thompson, Boyens, \& Poulton, 2011; World Health Organization, 2012).

Consequently, it is important to understand how individuals perceive and process information relating to the health benefits and outcomes associated with their oral self-care, not least because this can inform interventions designed to promote oral health. Previous studies have explored how oral health promotion can be encouraged by individual comparing their oral health behaviours to others, for example, via; positive role models (Hurling, et al., 2013), or using social or group based norms in terms of the theories of reasoned action or the theory of planned behaviour (e.g. Defranc, et al. 2008; Dumitrescu, Dogaru, Duta, \& Manolescu, 2014; Trubey, Moore, Chestnutt, 2015). These approaches share the assumption that individuals make decisions based on advice from others that provide a single and/or absolute reference point (e.g. a group norm) about the behaviours required to maintain good oral hygiene (i.e. tooth-brushing for a recommended length of time or number of occasions per day).

In this article, a specific hypothesis (the tooth-brushing rank hypothesis) is proposed and tested. The hypothesis states that individuals compare themselves to others when making judgments about their tooth-brushing behaviour, a hypothesis that contrasts with the approaches that make comparisons against a single or absolute reference point. The rank-based hypothesis 
proposes that individuals make judgments about tooth-brushing behaviour based on where their present behaviour falls within a social ranking system, and that comparisons are made with respect to numerous reference points. This tooth-brushing rank hypothesis derives from a theoretical formulation proposed by Maltby, Wood, Vlaev, Taylor, and Brown (2012) that integrates (1) evolutionary theories of rank-sensitivity to explain why individuals are motivated to make social rank judgments about positive health behaviours; and (2) the Decision-bySampling (DbS) model of judgment and decision-making (Stewart, Chater \& Brown, 2006) to explain how individuals make social rank judgments relating to positive health behaviours.

The first consideration is that individuals are motivated to make multiple comparisons through social-ranking. Evolutionary theory suggests that, within social systems a strong motivation emerges in which members compare themselves against one another to determine social rank (Sapolsky, 2004; Wilson, 1975). Ranked position within a social hierarchy is associated with the investments made in health systems (e.g. appearance, adrenocortical, cardiovascular, dietary, reproductive, and immune systems), that influence physical growth and social competition, which, in turn, influence survival and reproduction (Sapolsky, 2004). Therefore, individuals have a proclivity to compare their health to that of people around them to determine the social rank, and consequently make assessments about the benefits of engaging in specific health behaviours based on those comparisons. Therefore, individuals are motivated to select positive health behaviours because they judge this will ensure their survival and affirm or improve their social status within a social system (Sapolsky, 2004).

The second consideration is how individuals within a given social rank make rank-based judgments. According to the DbS model, the judgment of a quantity (e.g., tooth-cleaning duration) is context-dependent and determined by the relative ranked position of that quantity within the context of other available information. The DbS model suggests that, rather than one 
comparison being made against a single figure, individuals make comparisons against a sample containing multiple individuals. A person compares their own behaviour with that of each other person within their sample, deciding whether each other person engages in that behaviour more or less than they do. The person then is able to determine their rank within this perceived distribution. The best example of how such a quantitative based judgment might apply to oral care would be tooth-brushing duration, as this presents a degree of quantitative variation in the time engaged in the behaviour. In this example, rather than simply making a comparison between their own tooth-brushing duration and an NHS recommendation, two individuals might consider their behaviour within their peer group, and rank themselves relative to those individuals. Therefore, those two individuals may clean their teeth for the same duration, but the person who perceives their oral behaviour to rank higher among that of their peers may imagine themselves to be gaining greater benefits from their own behaviour, due to this favorable comparison. When this is considered in terms of promoting tooth-brushing, encouraging individuals to make comparisons against a single reference point (e.g. NHS guidelines) may not produce the greatest possible change in tooth-brushing, as it may not target, or encompass, individuals' natural propensity for making multiple-comparison judgments.

This rank-based approach is consistent with experimental research showing sensitivity to rank in judgments of psychophysical data (Parducci \& Perrett, 1971; Riskey, Parducci, \& Beauchamp, 1979), and also health, including exercise (Maltby et al., 2012) and alcohol consumption (Wood, Brown, \& Maltby, 2012). Most commonly this research has explored how quantitative measures of behaviour (e.g. amount of alcohol drunk or exercise undertaken within a specific timeframe) are influenced by social rank. In terms of tooth-brushing, a central recommendation is for individuals to spend two minutes cleaning their teeth with fluoride toothpaste. Therefore, considering how social rank may influence tooth-brushing duration would 
be a reasonable undertaking. Moreover, such an approach could form the basis of a low-cost, low-resource oral health intervention.

Accordingly, the present research examined the tooth-brushing rank hypothesis by focusing on the tooth-brushing duration in three studies, as tooth brushing duration will provide a good indication of how such a quantitative based judgment might apply to oral care. Study 1 examined the hypothesis from an attitudinal perspective, and whether perceptions of health benefits related to tooth-brushing duration could be predicted from individuals' ranking of their tooth-brushing behaviour, while controlling for other relevant variables (including self-perceived distance from a mean tooth-brushing duration). Study 2 examined the hypothesis from a cognitive judgment perspective, and whether experimentally manipulating rank positions can influence perceptions of the health benefits associated with tooth-brushing duration. Study 3 examined from a behaviour perspective, and whether information relating to rank position can influence self-reported tooth-brushing duration.

\section{Study 1}

Study 1 examined whether perceptions of the health benefits related to tooth-brushing among young adults can be predicted from the ranking of each person's tooth-brushing duration.

\section{Method}

\section{Participants.}

One hundred and twenty nine undergraduate students (40 males, 89 females) aged 18-34 years $(M=19.71, S D=2.6)$ completed a three-section questionnaire. Participants were volunteers, recruited using a research participation scheme, run within a single degree programme, and the study was completed via an online survey system. Undergraduate students are a useful sample to study as they are beginning to make health behaviour choices under their own volition, rather than necessarily following parental guidance at home. Arequired sample size of $n=>92$ was 
determined for multiple regression using GPower-3 (Faul, Erdfelder, Lang, \& Buchner, 2007), with five predictor variables (sex, age, self-report tooth-brushing duration, mean assessment, and rank assessment), with a power level of.8, probability level at .05, and an anticipated medium effect size $\left(f^{2}=.15\right)$, consistent with previous findings (Maltby et al., 2012; Wood et al., 2012).

\section{Measures.}

Respondents completed a three-section questionnaire. In Section 1, participants reported how long they spent brushing their teeth (in seconds). This assessment, as with the other studies reported in this paper, was self-report. While self-report measures of oral behaviour are not always reliable or valid and require careful calibration (e.g. Gil et al., 2015; Honghu, et al., 2010), there is nevertheless good evidence that self-reports of teeth-cleaning behaviour exhibit ‘acceptable’ clinical validity (kappa = .61; Jamieson, Thomson, \& McGee, 2004) and present a useful proxy or indicator of oral disease and risk behaviours, particular when clinical health assessments are unavailable (Gil, et al. 2015; Miller, Eke, \& Schoua-Glusberg, 2007). In Section 2, respondents answered six questions related to perceptions of the health benefits of their current tooth-brushing duration. Items were derived from advice from the NHS on "How to keep one's mouth clean", which highlights factors including maintaining oral health, and avoiding plaque, bacteria, gum disease, tooth decay, and cavities (National Health Service, 2013). The items were (all prefixed with "to what extent do you think the current time you spend brushing your teeth keeps....”) (1) “your teeth and mouth healthy”, (2) "you from developing gum disease", (3) "you from developing tooth decay", (4) "you from developing tooth decay cavities" (5) "the bacteria from building up around your teeth and mouth", and (6) "plaque from building up". All items were scored on a ten-point scale from 1 [Not at all] to 10 [Very much so]. In Section 3, participants answered eleven questions about their beliefs regarding the distribution of other adults' (of the same sex and age as the respondent) average tooth-brushing duration in the UK. 
Social Ranking Effects on Tooth-brushing.

Each question required participants to complete the "how many" part of the question within the following template: “In terms of time spent brushing one’s teeth, the top $x \%$ of individuals from the UK adult population (of the same sex and age as you) brush their teeth for more than *how many* seconds per session on average?”, with $x$ respectively taking values of $10,20,30,40,50$, 60, 70, 80, and 90 .

\section{Ethics.}

Ethical approval was obtained from an university ethics committee and complied with the British Psychological Society Code of Ethics. Participants provided consent via the first page of the electronic survey. The consent form contained statements regarding the nature of the study, anonymity, withdrawal, and the intended use, storage, and disposal of data.

\section{Results}

To demonstrate the utility of using the six questions in Section 2 as a summed measure of the perceived health benefits of tooth-brushing duration, reliability and validity estimates were produced. The Cronbach’s alpha (Cronbach, 1951) was $\alpha=.96$, exceeding the acceptable criteria of .70 (Kline, 1986). A maximum likelihood factor analysis was used to assess structural validity, with the emergence of a single factor (84.14\% of the variance) underlying the responses to the items, and this was supported by a parallel analysis (Zwick \& Velicer, 1986) in which the second eigenvalue $(5.05, .35)$ failed to exceed the second of the three mean eigenvalues $(1.30,1.16)$. These reliability and validity findings support the decision to form a single scale from the three items.

The participants' own tooth-brushing duration ranged from 10 to 600 seconds per session $(M=136.20, S D=106.46$, Median=120, interquartile range $=67-155$ seconds $)$. Table 1 shows the range, interquartile range, mean and median tooth-brushing duration, in seconds per session, as 
estimated by our participants for the top 10, 50 and 90 percent of the population. These statistics show that participants predict a decrease in the estimated average tooth-brushing duration from the top 10 percent to the top 90 percent of the population. However, the ranges in this table show that estimates within each category vary widely across participants, and that the estimates overlap across categories. This indicates that, within our sample, individuals have very different perceptions about tooth-brushing duration undertaken by the UK adult population across the distribution.

\section{INSERT TABLE 1}

A standardized multiple regression was performed to determine whether perceived rank predicts participants' perceptions of the health benefits of their current tooth-brushing duration. This analysis controlled for sex and age (as both variables are related to health practices; Deeks, Lombard, Michelmore \& Teede, 2009), actual tooth-brushing duration, and the extent to which each individual's actual tooth-brushing duration deviates from their estimate of the mean toothbrushing duration within the population (individuals in the UK of the same sex and age as themselves). Rank was calculated by determining where (in terms of $10^{\text {th }}, 20^{\text {th }}, .$. , or $90^{\text {th }}$ percentile) each individual's tooth-brushing duration ranked within their own subjective distribution of tooth-brushing duration within the population. Similarly, the perceived distance from the mean was calculated by comparing each participant's tooth-brushing duration against the mean for their subjective distribution between the 10th to 90th percentiles. The variables entered into the multiple regression significantly predicted perceived health benefits $(R=.35$, $R^{2}=.13$, Adjusted $\left.R^{2}=.09 ; F_{5,123}=3.52, p=.005\right)$. Both the participants' reported tooth-brushing duration and their perceived rank within the subjective distribution of the UK population accounted for unique variance in the perceived health benefits. Thus self-perceptions of tooth- 
brushing rank predicts unique variance (to a medium effect size) in the perceived health benefits of tooth-brushing, when controlling for other variables.

\section{INSERT TABLE 2}

\section{Study 2}

Study 2 tested whether manipulating the rank position causally influenced the participantperceived health benefits of tooth-brushing duration.

\section{Method}

\section{Participants.}

One hundred and one undergraduates (29 males; 72 females) aged 18-25 years $(M=19.93$; $S D=1.7$ ) took part in the study. Recruitment and ethical approval followed the same approach as in Study 1, but the study was administered in a different academic year. As the benchmarks provided by Cohen (1988) defining small, medium and large effects using eta-squared in ANOVA study designs are not suitable for studies that use constrained populations or repeated measures (Lakens, 2013; Olejnik \& Algina, 2003), we followed the recommendation to use effect size calculations ( $d=.2$ [small], .5[medium], .8[large]) derived from comparison of means for $t$-tests (Lakens, 2013). The results from GPower-3 (Faul et al., 2007) suggested a total sample of $n=52$ was required to detect a significant difference at the $p<.05$ level of significance (twotailed), to achieve a power of .8, and to detect a difference between the groups of a large effect size. A large effect size was chosen based on findings from other studies using a similar experimental method to evaluate the causal influence of perceived social rank on health behaviours (Maltby et al., 2012; Wood et al., 2012).

\section{Procedure.}

INSERT TABLE 3 
The procedure followed a previously used experimental rank research paradigm (Maltby et al., 2012; Wood et al., 2012). Respondents are randomly assigned to one of two experimental groups. Each participant is presented with one of two sets of 11 quantities of tooth-brushing duration. The distribution of tooth-brushing durations presented to respondents varied between the two experimental groups, and are illustrated in Table 3. These distributions are termed either “unimodal” or “bimodal” (e.g. Wood et al., 2012). Both groups saw an equal number of durations of tooth-brushing, ranging from 12 to 224s, with the average number of seconds in each distribution being 90s. This average figure was used as it was the midpoint of the interquartile range (60-120 seconds) and the closest (to the nearest 10 seconds) to the mean estimated duration of a tooth-brushing session for the top $50 \%$ of the UK's young adult population obtained from Study 1.

The two experimental groups had five durations in common; 36s, 64s, 90s, 116s, $144 \mathrm{~s}$. Three common points (36s, 90s, and $144 \mathrm{~s})$ have the same ranked position $\left(1^{\text {st }}, 6^{\text {th }}\right.$ and $\left.11^{\text {th }}\right)$ in both distributions. Two common points (64s and 116s) are in different rank positions (64s, $2^{\text {nd }}$ in the unimodal and $5^{\text {th }}$ in the bimodal groups; $116 \mathrm{~s}, 10^{\text {th }}$ in the unimodal and $7^{\text {th }}$ in the bimodal groups). If participants simply judged the health benefits of the five common tooth-brushing durations in isolation, then each tooth-brushing duration should receive a similar health value rating across both experimental groups due to being the same time durations. However, if judgments are based on rank, then only where the common point is equal in rank in both groups (36s [ranked 1st; common point 1], 64s [ranked 6th; common point 3] and 144s [ranked 11th; common point 5]) should there be similar health value ratings. Further, the 2nd common point (64s) should be seen as of less health value in the unimodal group (ranked 2nd, the second lowest rank) than in the bimodal group (ranked 5th). Similarly, the 4th common point (116s) should be 
seen as of more health value in the unimodal group (ranked 10th) than in the bimodal group (ranked 7th).

For each duration, participants were asked to make judgments about the health benefits of an individual who undertook that tooth-brushing duration. These judgments were based on the six health benefits statements in Study 1. Items were (with all items prefixed with "to what extent do you think the time the individual spends brushing their teeth keeps....”) (1) "their teeth and mouth healthy", (2) "them from developing gum disease", (3) "them from developing tooth decay", (4) "them from developing tooth decay cavities", (5) "the bacteria from building up around their teeth and mouth", and (6) "plaque from building up". All items were scored on a 1 [Not at all] to 10 [Very much so] scale.

\section{Results}

\section{INSERT TABLE 4}

As with Study 1, internal reliability estimates for the six questions across each common time point (36, 64, 90, 116, 144 seconds) suggested that the perceived health benefits items could be summed ( $\alpha>$.89). An interaction was tested using a 2(between: group) x 5(within: common point) ANOVA. There was a main effect of comparison point $\left(F_{4,99}=252.58, p<.001, \eta_{\mathrm{p}}{ }^{2}=.72\right)$ and the interaction between the experimental group and the comparison point was significant $\left(F_{4}\right.$, ${ }_{99}=15.28, p<.001, \eta_{\mathrm{p}}{ }^{2}=.13$ ), suggesting that the perceived health benefits associated with toothcleaning duration depended on the rank of that duration within each condition. Table 4 shows comparisons of the mean perceived health benefits of tooth-brushing for the unimodal and bimodal groups across each common point, reporting independent samples $t$-test, $p$-value and effect size (d) statistics.

\section{INSERT FIGURE 1}


The overall interaction is illustrated in Figure 1. Where the rank was the same in both groups at common points 1 (36 seconds, rank=1), 3 (90 seconds, rank=6) and 5 (144 seconds, rank=11), no significant differences were observed between the unimodal and bimodal groups in terms of overall perceived health benefits. However, common point 2 (64 seconds) was rated as significantly less beneficial to health (with a large effect size) in the unimodal group (where its rank was 2) than the bimodal group (where its rank was 5). Conversely, common point 4 (116 seconds) was rated as significantly more beneficial to health (with a medium effect size) in the unimodal group (where its rank was 10th) than the bimodal group (where its rank was 7th). It is important to note, in terms of the latter finding, that the calculation of sample size required the findings to be of a large effect size. Post-hoc analysis of the power of the result using GPower-3 predicted that power of this latter finding $(n=49 / 52, d=.5, p=.044$, one-tailed, as the direction was predicted a priori) to be .78 , below the acceptable criteria of .80 .

\section{Study 3}

Study 3 examined whether messages relating to the rank positions of tooth-brushing durations causally influenced tooth-brushing duration.

\section{Method}

Participants.

Participants were 222 undergraduate students (43 males, 179 females) aged 18-37 years $(M=19.66, S D=2.4)$. Figure 2 outlines the flow of participants through the study.

\section{INSERT FIGURE 2}

The sample was from a larger sample of 525 undergraduate students from multiple degree programmes (113 males, 412 females; $M$ age=19.83, $S D=2.8$ ) who answered a screening question of "On average, how long have you spent cleaning your teeth over the last five days per session?”. An additional 29 respondents withdrew at the screening stage or were excluded as 
they provided implausible responses to the screening question, e.g. cleaning one's teeth for 20 minutes.

Central to testing our hypothesis was a comparison of changes in tooth-brushing behaviour across two time-points following an experimental manipulation. GPower-3 (Faul et al., 2007) suggested a sample of $n=34$ was needed for a two-tailed within-participants mean comparison across two time-points with a power of .8 , and a medium-sized difference $(d=0.5)$ at the $p<.05$ level. No previous findings could be used to determine the expected effect size, and so this criterion was employed as the minimum at which findings are likely to be of practical significance (Cohen, 1988, Lipsey, 1998). With five participant groups, all $n>=34$, meant a sample size of at least $n=170$. Participants were included in the study if they reported a lower tooth-brushing duration than the recommended two-minute threshold duration. To ensure recruitment and retention of sufficient individuals would be recruited, and meet screening requirements, a series of data collections was completed in three separate time periods (within two years), obtaining samples of $n=200, n=107$ and $n=218$. No significant difference $(F(2$, $522)=.41, p=.662$ ) was found for tooth-brushing duration across these samples (Sample 1, $M=108.24$ seconds, $S D=72.54$; Sample 2, $M=111.39, S D=78.15$; Sample 3, $M=114.63$, $S D=74.56)$.

\section{Procedure.}

Respondents were randomly sequentially allocated to one of five participant groups

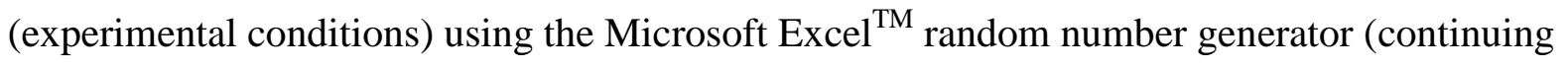
sequentially across the time-points). The first was a social rank judgment group. In this condition, participants were informed how their tooth-brushing behaviour compared to others in terms of a social rank distribution. The second group was a 'mean average' judgment group. Participants were informed how their tooth-brushing behaviour compared to others in terms of a 
group mean. The third group was an official single value judgment group. In this condition, participants were informed how their behaviour compared to NHS guidelines of 120 seconds of tooth-brushing. The fourth participant group was a first control group. In this condition, participants were informed of official NHS guidelines relating to healthy eating, but no mention was made of their own tooth-brushing behaviour. The fifth participant group was a second control group. Here no information was provided to the participants. Forty five participants were assigned to the social rank and the rank mean judgment experimental groups, and 44 participants were assigned to each of the three remaining groups. No significant difference found between the five groups for the baseline scores for tooth-brushing duration $(F(4,209)=.44, p=.78)$.

The study lasted four days. On the first day (Time 1), participants were asked to report, via an electronic survey, the time (in seconds) they cleaned their teeth the night before. Then, over the next two days, the participants received one message per day. These messages were the same on each day but different for participants allocated to each of five different experimental conditions. The messages were sent by email. In the social rank judgment condition, the messages informed participants how the duration of their tooth-brushing ranked compared to the first sample of 200 respondents who had completed the survey (this sample was used so that all participants received messages making comparisons within the same distribution). Participants were reminded of their previous self-report of teeth-cleaning duration, and were told where that positioned them within the peer distribution (e.g. 'this places you in the bottom X\%'). Those in the mean average judgment condition were informed how the duration of their tooth-brushing compared to the mean ( $M=108$ seconds) of the first 200 respondents in the sample. Those in the official single value judgment condition were reminded of their self-reported tooth-brushing duration and the NHS guideline figure. Participants in one of two control conditions were informed of their tooth-brushing duration and provided with NHS guidelines relating to eating 
five portions of fruit or vegetables a day (National Health Service, 2014). Participants in the second control condition were informed of their self-reported tooth-brushing duration only. At the end of the email, participants were asked to confirm that they had received and read the email by completing a quick online survey linked to the email. On the final day (Time 2), the participants were asked to report, via an electronic survey, the duration (in seconds) that they had cleaned their teeth the night before. On completion of the study, participants were given either a small monetary payment or a book token to the value of $£ 10$ or credit as part of a university experimental participation scheme.

\section{Results}

Of the original 222 participants, 191 respondents completed the study. Figure 2 shows retention at allocation, follow-up, and analysis stages. The final sample sizes of the participant groups were as follows: social rank judgment $n=37$, mean average judgment $n=39$, official single value judgment $n=37$, first control group $n=42$, and second control group $n=36$. The attrition rate for these groups did not differ significantly $\left(X^{2}(4)=4.60, p=.331\right)$.

An ANOVA revealed a difference effect between the time-points $\left(F_{4,186}=34.30, p<.001\right.$, $\left.\eta_{\mathrm{p}}{ }^{2}=.16\right)$ and a significant interaction between the experimental group and time-point $\left(F_{4}\right.$, ${ }_{186}=4.13, p=.003, \eta_{\mathrm{p}}{ }^{2}=.08$ ), suggesting that changes in tooth-brushing duration were related to the experimental conditions. Table 5 shows the mean comparisons, dependent sample $t$-test, $p$ value and effect size $(d)$ for tooth-brushing duration for Time 1 and Time 2 by each experimental group. A statistically significant difference was found for social rank, mean average and official single value judgment groups, with the differences between Time 1 and Time 2 being of a small effect size for social rank, and a medium effect size for the latter two groups. 


\section{Discussion}

The results show that individuals’ perceptions of the health benefits of brushing their teeth for two minutes, and the duration of their own teething cleaning, are influenced by how their toothbrushing duration ranks in comparison with the tooth-brushing duration of others. These findings present initial support for the tooth-brushing rank hypothesis.

How the perceptions of the health benefits of a given tooth-brushing duration are influenced by rank is shown in two complementary ways. Study 1 demonstrates that individuals' perceptions of the health benefits of their own current tooth-brushing duration are predicted by their own tooth-brushing duration and the perceived rank of that duration (to a medium effect size) within their perception of the distribution of tooth-brushing duration among the UK population, while controlling for sex, age, and distance of tooth-brushing duration from the mean tooth-brushing duration within the perceived distribution across the UK population. Study 2 demonstrates that perceptions of the health benefits of a given tooth-brushing duration can be experimentally manipulated by changing the ranking of that duration. The sizes of effects across these studies range from medium (Study 1 and common point 4 in Study 2) to large (common point 2 in Study 2), noting that in the former case in terms of point 4 in Study 2 this finding does not have acceptable power. Study 3 suggests the length of time individuals spend cleaning their teeth can be increased by using an intervention that provides information about how they rank in comparison to their peers in terms of tooth-brushing duration. The effect size was small, however, and by this criterion, this approach was not as effective as other comparison methods, whereby individuals were required to judge their own behaviour against the average of their peers or against national health guidelines. The effect sizes for these latter two conditions were medium, suggesting that the social rank comparison is the least effective of the three methods. 
A similar caveat applies to the finding regarding common point 4 in Study 2, which falls short of the required power. This may reflect a ceiling effect, with perceptions that the health benefits of brushing one's teeth declines with increasing duration. However, while the perceived health benefits of brushing one's teeth may decline for periods that exceed 2 minutes, this does not negate the findings from the present research showing that the decision to brush one's teeth for less than 2 minutes can be influenced by social rank information.

Indeed, overall the findings provide empirical support for a tooth-brushing social rank hypothesis as derived from evolutionary accounts of the motivation to produce physiologically healthy systems and the DbS model of cognitive judgments. Specifically, the findings suggest that social rank can influence perceptions of the benefits of specific tooth-cleaning behaviours and that this can influence actual tooth-cleaning behaviour. Study 3 suggests this influence on behaviour is rather small and larger effects sizes were obtained for guidance based information about normative behaviours and official advice. These findings will help the design of interventions aimed at improving teeth-cleaning, and suggest that an effective implementation message might emphasize both rank positions and normative information. Including rank as an information source would be consistent with "nudge style” interventions that use multiple, rational and automatic cues to influence decision-making (Dolan et al., 2012). The present findings also indicate that rank-based methods can provide an alternative technique to promote behaviour change in the event that interventions based on other comparison methods fail. There are three considerations to the study. The first of these is our reliance on self-report measures of behaviour. Though self-report measures often show clinical validity, we nevertheless recognise their shortcomings. For example, in Study 1, particular motivations (such as relative pleasure) may distort the perceived time spent brushing one teeth. Similarly, in Study 3, the differences in self-reported teeth cleaning may represent changes in reported behaviour or beliefs concerning 
appropriate behaviour rather than actual behaviour change (though changes in beliefs about appropriate behaviours may act as intervention in itself). Further research should employ objective or independent assessments of tooth-brushing, such as smart tooth-brushes, to assess the effectiveness of information designed to effect behaviour change. Second, we have attended to social-cultural influences on teeth-cleaning behaviour, which may place different weight on the importance of teeth-cleaning and comparisons with the behaviours of others (for example, in families where teeth-cleaning is not valued). Third, participant recruitment in Study 3 had to be staged so as to obtain sufficient participants to satisfy a power analysis. Clearly, a random, controlled trial in which all participants started the study at the same time, and completed it over the same period, would be preferable.

In summary, the present findings reveal the relationship between how an individual perceives how his or her tooth-brushing duration ranks relative to other people and the duration for which they clean their teeth. Together, the results support a tooth-brushing rank hypothesis, and introduce a theoretical and empirical approach for exploring how social ranks influence perceptions and behaviour concerning tooth-brushing duration. 


\section{References}

Attin, T. \& Hornecker, E. (2005). Tooth brushing and oral health: how frequently and when should tooth brushing be performed? Oral Health and Preventive Dentistry, 3(3): 135140.

Broadbent, J.M., Thomson, W.M., Boyens, J.V. \& Poulton, R. (2011). Dental plaque and oral health during the first 32 years of life. Journal of the American Dental Association, 142(4), 415-426.

Cohen, J. (1988). Statistical power analysis for the behavioral sciences (2nd ed.). Hillsdale, NJ: Erlbaum.

Cronbach, L.J. (1951). Coefficient alpha and the internal structure of tests. Psychometrika, 16(3), 297-334. doi:10.1007/BF02310555.

Deeks, A., Lombard, C., Michelmore, J. \& Teede, H. (2009). The effects of gender and age on health related behaviours. BMC Public Health, 9: 213. doi: 10.1186/1471-2458-9-213.

Defranc, A., Van den Broucke, S., Leroy, R., Hoppenbrouwers, K., Lesaffre, E., Martens, L., Debyser, M. \& Declerck, D. (2008). Measuring oral health behaviour in Flemish health care workers: an application of the Theory of Planned Behaviour. Community Dental Health, 25(2), 107-114. Doi: 10.1922/CDH_2120VandenBroucke08 .

Dolan, P., Hallsworth, M., Halpern, D., King, D., Metcalfe, R., \& Vlaev, I. (2012). Influencing behavior: The mindspace way. Journal of Economic Psychology, 33(1), 264-277. doi:10.1016/j.joep.2011.10.009.

Dumitrescu, A. L. Dogaru, B.C., Duta, C. \& Manolescu, B. N. (2014). Testing five socialcognitive models to explain predictors of personal oral health behaviours and intention to improve them. Oral Health and Preventive Dentistry, 12(4), 345-355. 
Faul, F., Erdfelder, E., Lang, A.G., \& Buchner, A. (2007). G*Power 3: a flexible statistical power analysis program for the social, behavioral, and biomedical sciences. Behavioural Research Methods, 39(2), 175-191. doi:10.3758/BF03193146.

Gil, G. S., Morikava, F. S., Santin, G. C. , Pintarelli, T. P., Fraiz, F. C. \& Ferreria, F. M. (2015). Reliability of self-reported toothbrushing frequency as an indicator for the assessment of oral hygiene in epidemiological research on caries in adolescents: a cross-sectional study. BMC Medical Research Methodology, 15(14). doi: doi:10.1186/s12874-015-0002-5.

Hurling R., Claessen, J.P., Nicholson, J., Schäfer, F., Tomlin, C.C., \& Lowe, C.F. (2013). Automated coaching to help parents increase their children's brushing frequency: an exploratory trial. Community Dental Health, 30(2), 88-93.

doi:10.1922/CDH_3035Hurling06.

Jamieson, L. M., Thomson, W. M., \& McGee, R. (2004). An assessment of the validity and reliability of dental self-report items used in a National Child Nutrition Survey. Community Dentistry and Oral Epidemiology, 32(1), 49-54. doi:10.1111/j.16000528.2004.00126.X

Kline, P. (1986). A handbook of test construction: Introduction to psychometric design. New York, NY: Methuen.

Lakens, D. (2013). Calculating and reporting effect sizes to facilitate cumulative science: a practical primer for t-tests and ANOVAs. Frontiers in Psychology, 4, 863. doi:10.3389/fpsyg.2013.00863.

Lipsey, M.W. (1998). Design sensitivity: Statistical power for applied experimental research. In L. Bickman \& D.J. Rog (Eds.), Handbook of applied social research methods (pp. 3968). Thousand Oaks, CA: Sage. 
Social Ranking Effects on Tooth-brushing.

Liu, H., Maida, C. A., Spolsky, V. W., Shen, J., Li, H., Zhou, X., \& Marcus, M. (2010).

Calibration of self-reported oral health to clinically determined standards. Community Dentistry and Oral Epidemiology, 38(6), 527-539. doi:10.1111/j.16000528.2010.00562.x

Maltby, J., Wood, A.M., Vlaev, I., Taylor, M.J., \& Brown, G.D.A. (2012). Contextual effects on the perceived health benefits of exercise: The exercise rank hypothesis. Journal of Sport \& Exercise Psychology, 34(6), 828-841.

Miller, K., Eke, P. I., \& Schoua-Glusberg, A. (2007). Cognitive Evaluation of Self-Report Questions for Surveillance of Periodontitis. Journal of Periodontology, 78(7), 1455-1462. doi: 10.1902/jop.2007.060384.

National Health Service (2013, December 2). How to keep your teeth clean. Retrieved from http://www.nhs.uk/Livewell/dentalhealth/Pages/Teethcleaningguide.aspx.

National Health Service (2014). 5 A DAY. Retrieved from http://www.nhs.uk/Livewell/5ADAY/Pages/5ADAYhome.aspx (27th November, 2014).

Newton, T.L., Bane, C.M., Flores, A., \& Greenfield, J. (1999). Dominance, gender, and cardiovascular reactivity during social interaction. Psychophysiology, 36(2), 245-252. doi:10.1017/S0048577299971986.

Olejnik, S., \& Algina, J. (2003). Generalized eta and omega squared statistics: Measures of effect size for some common research designs. Psychological Methods, 8(4), 434-447. doi:10.1037/1082-989X.8.4.434.

Parducci, A., \& Perrett, L.F. (1971). Category rating scales: Effects of relative spacing and frequency of stimulus values. Journal of Experimental Psychology Monograph, 89(2), 427-452. doi:10.1037/h0031258. 
Social Ranking Effects on Tooth-brushing.

Riskey, D.R., Parducci, A., \& Beauchamp, G.K. (1979). Effects of context in judgments of sweetness and pleasantness. Perception \& Psychophysics, 26, 171-176. doi:10.3758/BF03199865.

Sapolsky, R.M. (2004). Social status and health in humans and other animals. Annual Review of Anthropology, 33, 393-418. doi:10.1146/annurev.anthro.33.070203.144000.

Sasahara, H. \& Kawamura, M. (2000). Behavioral dental science: the relationship between tooth-brushing angle and plaque removal at the lingual surfaces of the posterior teeth in the mandible. Journal of Oral Science, 42(2), 79-82.

Stewart, N., Chater, N., \& Brown, G.D.A. (2006). Decision by sampling. Cognitive Psychology, 53(1), 1-26. doi:10.1016/j.cogpsych.2005.10.003.

Trubey, R. J., Moore, S. C. \& Chestnutt, I. G. (2015). The association between parents' perceived social norms for toothbrushing and the frequency with which they report brushing their child's teeth. Community Dental Health, 32(2), 98-103. Doi: 10.1922/CDH_3512Trubey06.

Wilson, E.O. (1975). Sociobiology: The new synthesis. Cambridge, MA: Belknap Press of Harvard University Press.

Wood, A.M., Brown, G.D.A., \& Maltby, J. (2012). Social norm influences on evaluations of the risks associated with alcohol consumption: Applying the rank-based decision by sampling model to health judgments. Alcohol and Alcoholism, 47(1), 57-62. doi:10.1093/alcalc/agr146.

World Health Organization, Geneva (2012, April). Oral health. Fact sheet $N^{\circ} 318$. Retrieved from http://www.who.int/mediacentre/factsheets/fs318/en/. 
Zwick, W.R., \& Velicer, W.F. (1986). Comparison of five rules for determining the number of components to retain. Psychological Bulletin, 99(3), 432-442. doi:10.1037/00332909.99.3.432. 
Table 1

Range, interquartile range and mean and median scores for estimated average duration of tooth-brushing session (in seconds) for the top 10, 50 and $90 \%$ of the UK young adult population.

\begin{tabular}{lllcc}
\hline & Range (Minimum-Maximum) & Interquartile range & Mean & Median \\
\hline $10 \%$ & $30-450$ & $90-167.50$ & 140.28 & 725.00 \\
$50 \%$ & $8-300$ & $60-120$ & 88.52 & 35.00 \\
$90 \%$ & $0-180$ & $20-60$ & 46.67 & 35.00 \\
\hline
\end{tabular}




\section{Table 2}

Standardized multiple regression analysis for perceived health benefits of tooth-brushing, with sex, age, duration of teeth brushing, difference from perceived mean and perceived rank used as predictor variables.

\begin{tabular}{|c|c|c|c|c|}
\hline & $B$ & $\beta$ & $t$ & $p$ \\
\hline Sex & .15 & .04 & .41 & 679 \\
\hline Age & .52 & .02 & .26 & .799 \\
\hline Self-reported teeth cleaning duration & .05 & .44 & 2.15 & .034 \\
\hline Difference from mean of subjective distribution of UK population's teeth cleaning duration & -.05 & -.40 & -1.65 & .101 \\
\hline Rank within subjective distribution of UK population's teeth cleaning duration & .15 & .36 & 2.89 & .004 \\
\hline
\end{tabular}




\section{Table 3}

Duration of tooth-brushing per session (in seconds) presented to both experimental groups, with common points bolded and underlined.

\begin{tabular}{|c|c|c|c|c|c|c|c|c|c|c|c|c|c|c|c|c|c|}
\hline Common point & 1 & & & & 2 & & & & 3 & & & & 4 & & & & $\underline{5}$ \\
\hline Unimodal & $\underline{36}$ & & & & $\underline{64}$ & 72 & 78 & 84 & $\underline{90}$ & 96 & 102 & 108 & $\underline{116}$ & & & & $\underline{144}$ \\
\hline Bimodal & $\underline{36}$ & 42 & 48 & 56 & $\underline{64}$ & & & & $\underline{90}$ & & & & $\underline{116}$ & 124 & 132 & 138 & $\underline{144}$ \\
\hline
\end{tabular}




\section{Table 4}

Means and standard deviations for overall perceived health benefits relating to duration of tooth-brushing for five different "seconds per session", by unimodal and bimodal group.

\begin{tabular}{llcccccccc}
\hline & & \multicolumn{7}{c}{ Overall perceived health benefits } \\
Common point & Seconds & Unimodal rank & Bimodal rank & Unimodal & Bimodal & $\mathrm{t}$ & $p$ & $d$ \\
\hline 1 & 36 & $1^{\text {st }}$ & $1^{\text {st }}$ & $19.51(9.4)$ & $16.92(10.6)$ & 1.30 & .197 & .26 \\
2 & 64 & $2^{\text {nd }}$ & $5^{\text {th }}$ & $27.55(9.8)$ & $38.73(11.6)$ & -5.21 & .001 & 1.04 \\
3 & 90 & $6^{\text {th }}$ & $6^{\text {th }}$ & $43.41(11.2)$ & $41.38(11.5)$ & .898 & .372 & .18 \\
4 & 116 & $10^{\text {th }}$ & $7^{\text {th }}$ & $49.59(10.7)$ & $43.94(11.2)$ & 2.58 & .011 & .50 \\
5 & 144 & $11^{\text {th }}$ & $11^{\text {th }}$ & $51.30(11.4)$ & $51.6538(11.5)$ & -.153 & .878 & .04 \\
\hline
\end{tabular}




\section{Table 5}

Time 1 and Time 2 Mean, Standard Deviation, t-test, probability and effect size statistics for experimental conditions (Study 3).

\begin{tabular}{|c|c|c|c|c|c|c|}
\hline & $n$ & $\begin{array}{c}\text { Time } 1 \\
\text { Mean (SD) }\end{array}$ & $\begin{array}{c}\text { Time } 2 \\
\text { Mean (SD) }\end{array}$ & $t=$ & $p=<$ & $d=$ \\
\hline Social rank judgment & 37 & $59.78(35.08)$ & $72.38(39.64)$ & -2.86 & .007 & .47 \\
\hline Mean average judgment & 39 & $58.46(32.41)$ & 75.13 (40.19) & -3.78 & .001 & .60 \\
\hline Official single value judgment & 37 & $55.81(33.07)$ & $78.84(39.36)$ & -4.25 & .001 & .69 \\
\hline First control group & 42 & $60.36(32.82)$ & $63.21(36.02)$ & -.82 & .416 & .13 \\
\hline Second control group & 36 & $61.67(31.12)$ & $64.03(37.43)$ & -.57 & .572 & .09 \\
\hline
\end{tabular}




\section{Figure 1}

Perceived health benefits relating to ratings of individual durations of tooth-brushing for five different numbers of seconds per session.

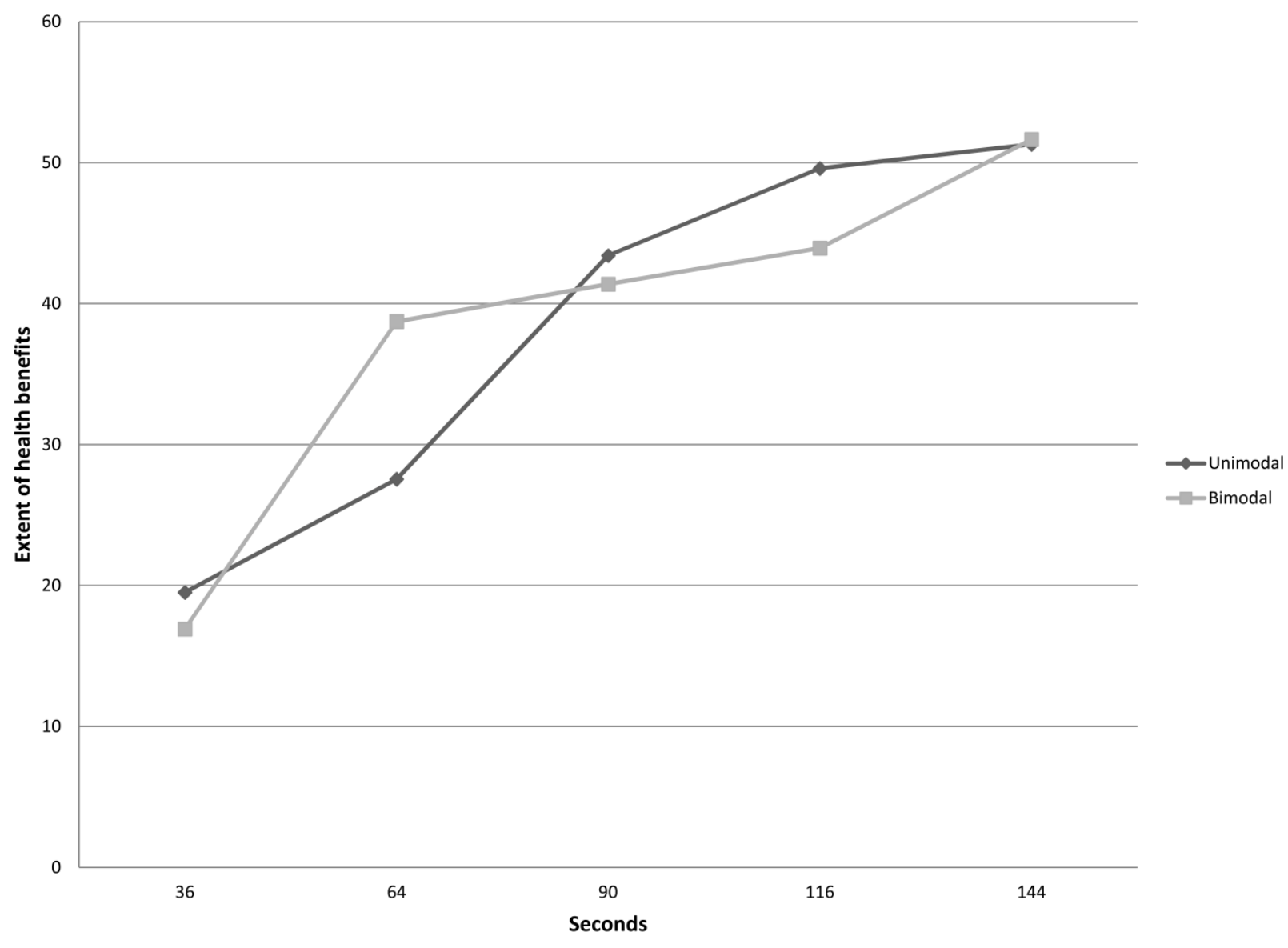




\section{Figure 2}

\section{CONSORT diagram to show flow of participants through Study 3.}

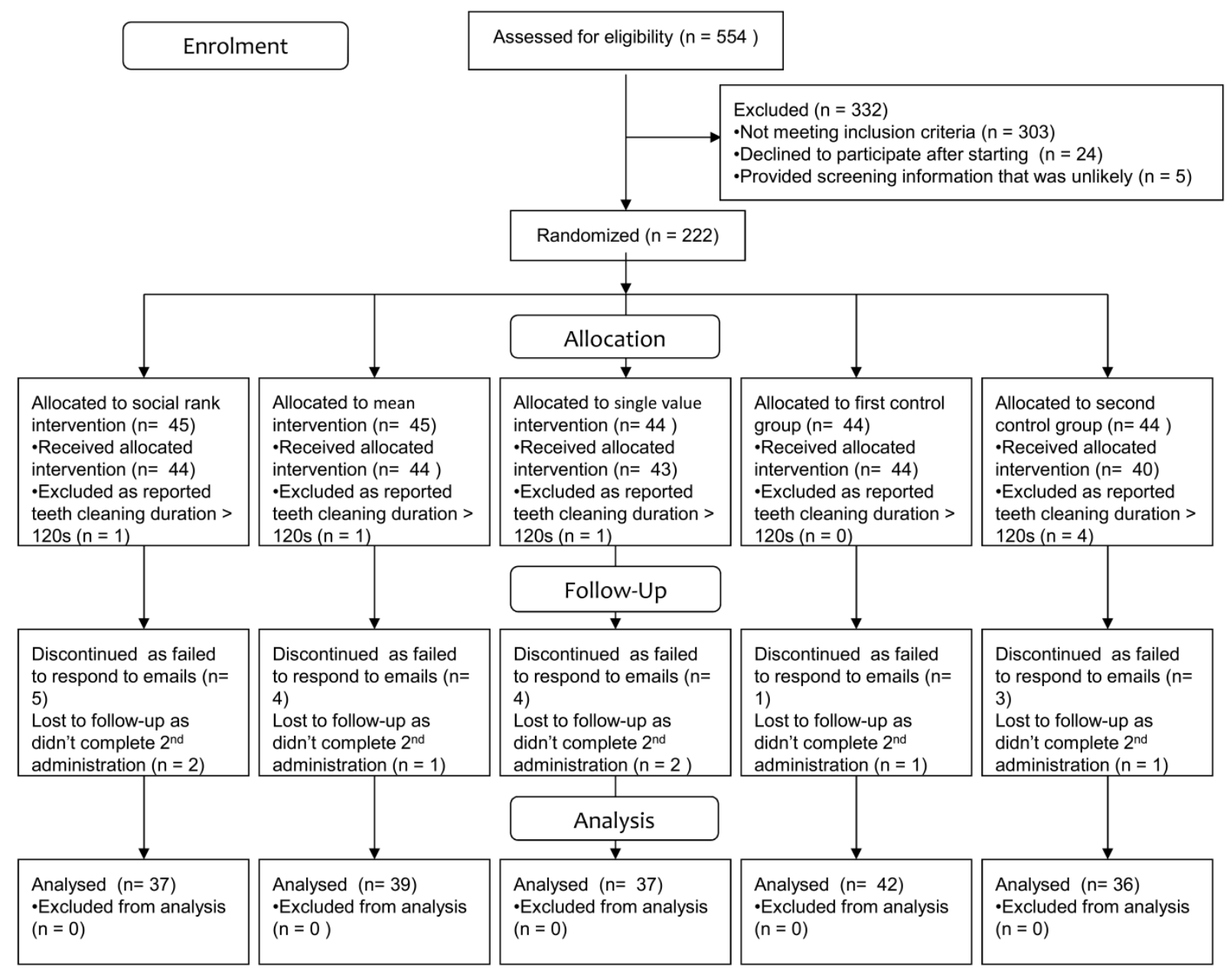


Table 1

Range, interquartile range and mean and median scores for estimated average duration of tooth-brushing session (in seconds) for the top 10, 50 and $90 \%$ of the UK young adult population.

\begin{tabular}{lllcr}
\hline & Range (Minimum-Maximum) & Interquartile range & Mean & Median \\
\hline $10 \%$ & $30-450$ & $90-167.50$ & 140.28 & 725.00 \\
$50 \%$ & $8-300$ & $60-120$ & 88.52 & 35.00 \\
$90 \%$ & $0-180$ & $20-60$ & 46.67 & 35.00 \\
\hline
\end{tabular}


Table 2

Standardized multiple regression analysis for perceived health benefits of tooth-brushing, with sex, age, duration of teeth brushing, difference from perceived mean and perceived rank used as predictor variables.

\begin{tabular}{|c|c|c|c|c|}
\hline & $B$ & $\beta$ & $t$ & $p$ \\
\hline Sex & .15 & .04 & .41 & .679 \\
\hline Age & .52 & .02 & .26 & .799 \\
\hline Self-reported teeth cleaning duration & .05 & .44 & 2.15 & .034 \\
\hline Difference from mean of subjective distribution of UK population's teeth cleaning duration & -.05 & -.40 & -1.65 & .101 \\
\hline Rank within subjective distribution of UK population's teeth cleaning duration & .15 & .36 & 2.89 & .004 \\
\hline
\end{tabular}


Table 3

Duration of tooth-brushing per session (in seconds) presented to both experimental groups, with common points bolded and underlined.

\begin{tabular}{|c|c|c|c|c|c|c|c|c|c|c|c|c|c|c|c|c|c|}
\hline Common point & 1 & & & & 2 & & & & 3 & & & & 4 & & & & $\underline{5}$ \\
\hline Unimodal & $\underline{36}$ & & & & $\underline{64}$ & 72 & 78 & 84 & $\underline{90}$ & 96 & 102 & 108 & $\underline{116}$ & & & & $\underline{144}$ \\
\hline Bimodal & 36 & 42 & 48 & 56 & 64 & & & & 90 & & & & 116 & 124 & 132 & 138 & 144 \\
\hline
\end{tabular}


Table 4

Means and standard deviations for overall perceived health benefits relating to duration of tooth-brushing for five different "seconds per session”, by unimodal and bimodal group.

\section{Overall perceived health benefits}

\begin{tabular}{lcccccccc} 
Common point & Seconds & Unimodal rank & Bimodal rank & Unimodal & Bimodal & t & $p$ & $d$ \\
\hline 1 & 36 & $1^{\text {st }}$ & $1^{\text {st }}$ & $19.51(9.4)$ & $16.92(10.6)$ & 1.30 & .197 & .26 \\
2 & 64 & $2^{\text {nd }}$ & $5^{\text {th }}$ & $27.55(9.8)$ & $38.73(11.6)$ & -5.21 & .001 & 1.04 \\
3 & 90 & $6^{\text {th }}$ & $6^{\text {th }}$ & $43.41(11.2)$ & $41.38(11.5)$ & .898 & .372 & .18 \\
4 & 116 & $10^{\text {th }}$ & $7^{\text {th }}$ & $49.59(10.7)$ & $43.94(11.2)$ & 2.58 & .011 & .50 \\
5 & 144 & $11^{\text {th }}$ & $1^{\text {th }}$ & $51.30(11.4)$ & $51.6538(11.5)$ & -.153 & .878 & .04
\end{tabular}


Table 5

Time 1 and Time 2 Mean, Standard Deviation, t-test, probability and effect size statistics for experimental conditions (Study 3).

\begin{tabular}{|c|c|c|c|c|c|c|}
\hline & $n$ & Time 1 & Time 2 & $t=$ & $p=<$ & $d=$ \\
\hline & & Mean (SD) & Mean (SD) & & & \\
\hline Social rank judgment & 37 & $59.78(35.08)$ & 72.38 (39.64) & -2.86 & .007 & .47 \\
\hline Mean average judgment & 39 & $58.46(32.41)$ & 75.13 (40.19) & -3.78 & .001 & .60 \\
\hline Official single value judgment & 37 & $55.81(33.07)$ & $78.84(39.36)$ & -4.25 & .001 & .69 \\
\hline First control group & 42 & $60.36(32.82)$ & $63.21(36.02)$ & -.82 & .416 & .13 \\
\hline Second control group & 36 & $61.67(31.12)$ & $64.03(37.43)$ & -.57 & .572 & .09 \\
\hline
\end{tabular}




\section{Figure 1}

Perceived health benefits relating to ratings of individual durations of tooth-brushing for five different numbers of seconds per session.

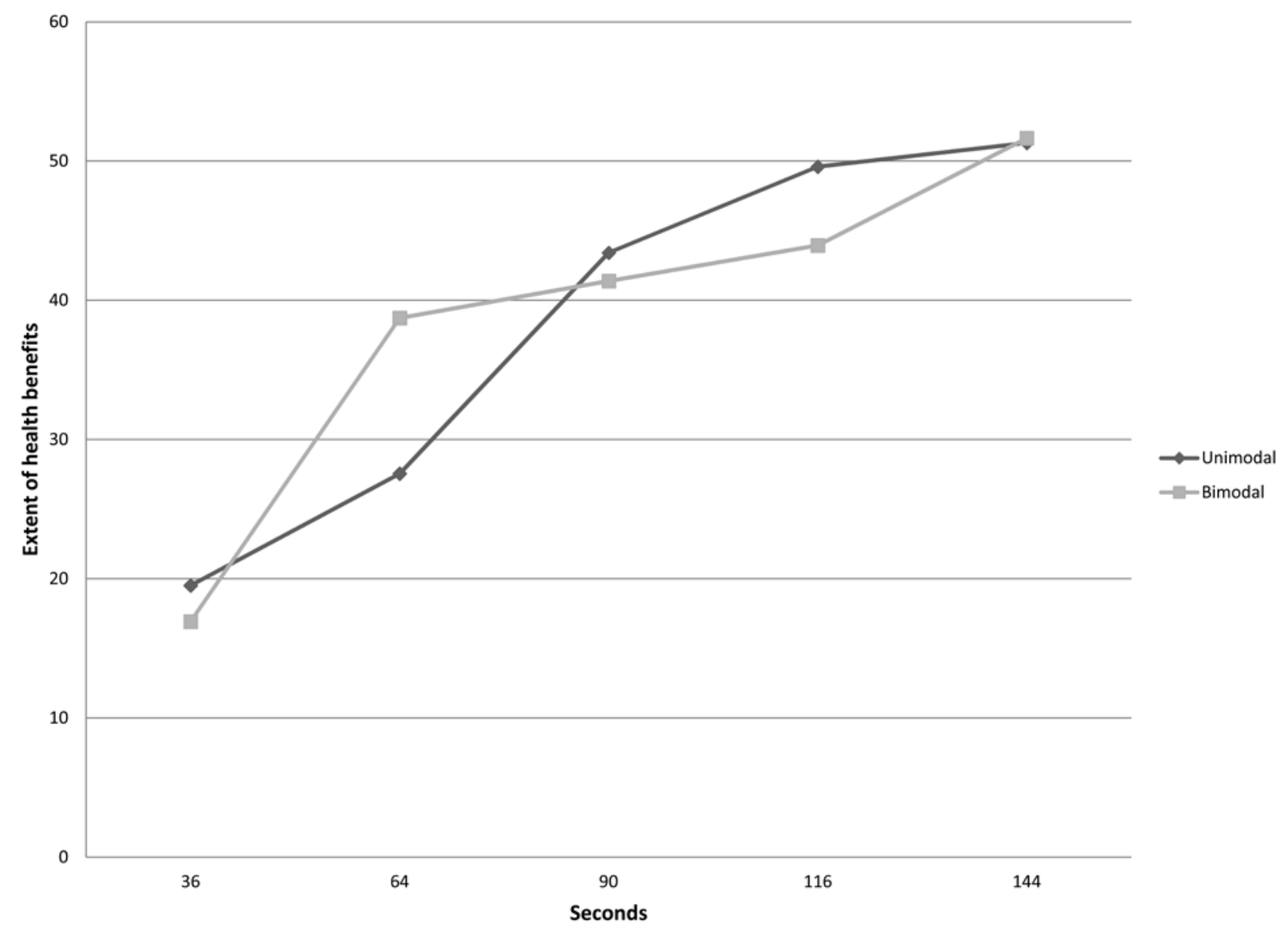


Figure 2

CONSORT diagram to show flow of participants through Study 3.

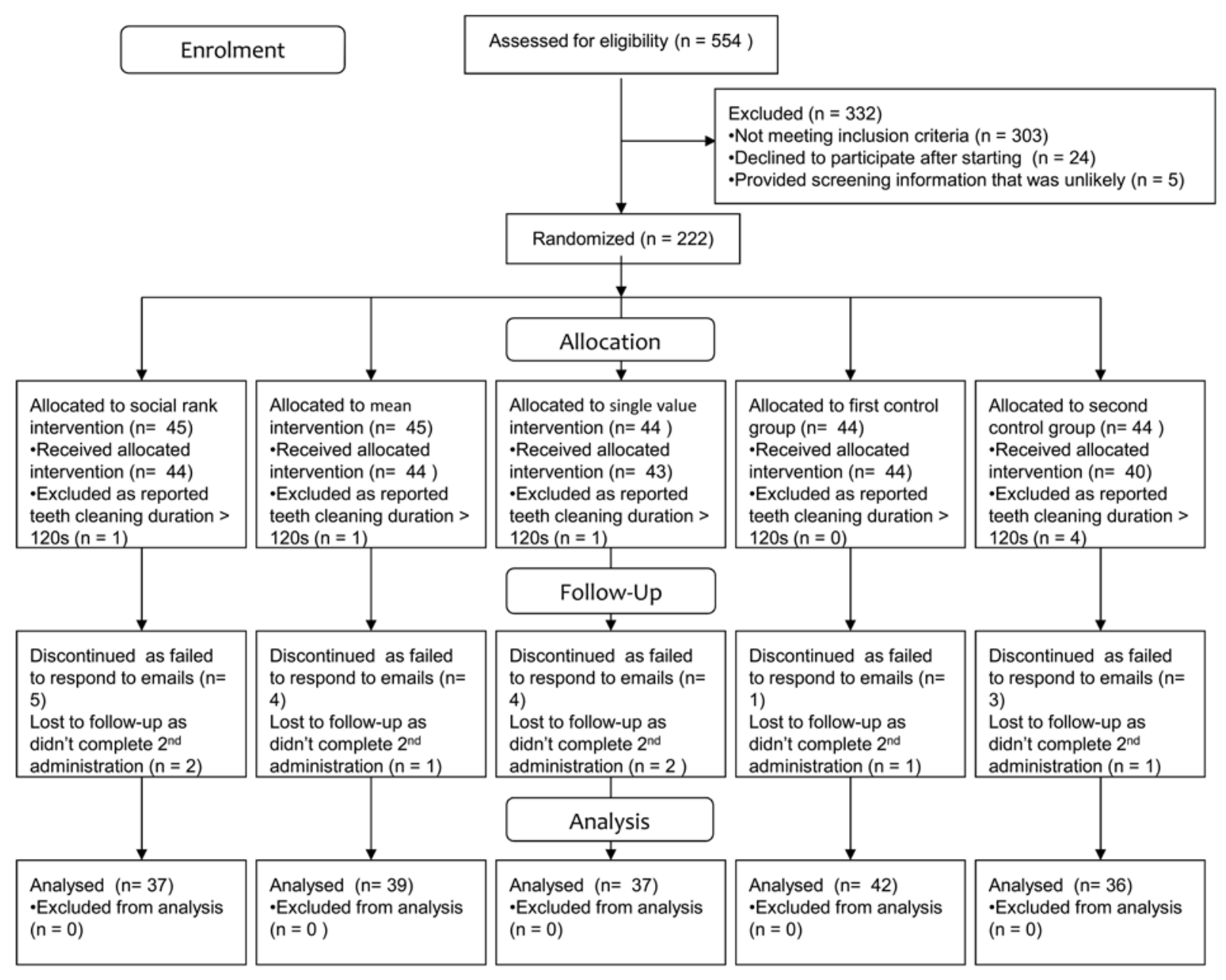

